Contents List available at RAZI Publishing

\title{
DETERMINATION THE MOST IMPORTANT OF HSE CLIMATE ASSESSMENT INDICATORS CASE STUDY: HSE CLIMATE ASSESSMENT OF COMBINED CYCLE POWER PLANT STAFFS
}

\author{
Reza Radmanfar'1, Maryam Rezayi ${ }^{1}$, Saghar Salajegheh' ${ }^{1}$, Vahid Arab Bafrani ${ }^{2}$
}

\author{
${ }^{1}$ Student of Industrial Engineering, Industrial Safety, University of Science and Arts of Yazd. \\ ${ }^{2}$ Student of Industrial Management., Production, University of Science and Arts of Yazd.
}

This is an open access article distributed under the Creative Commons Attribution License, which permits unrestricted use, distribution, and reproduction in any medium, provided the original work is properly cited

\section{ARTICLE DETAILS}

\section{Article History:}

Received 9 July 2017

Accepted 10 October 2017

Available online 6 November 2017

Keywords:

HSE

Culture,

Climate,

HSE

Commitment, Risk, Sustainable Development.
ABSTRACT

Doubtlessly, noting the growth of industry and the criticality of the environment at the present time and the significance of protecting and preserving the resources to achieve the sustainable development, establishing the appropriate cultural mechanisms which can be able to confront the probable problems rationally besides understanding the biological and human resources for achieving the goals of sustainable development and establish matching with the conditions is so necessary. Today, the subject of HSE in the industry and creating its relevant cultural context in the developing countries is significant and it is necessary to assess its position at the organizational level in several sessions. Assessing the climate of HSE in an organization can depict a realistic picture of the staff understanding of the subject of HSE and their duties. The purpose of carrying out this study is to identify the main assessing factors of the climate of HSE in an organization and studying one of the industrial units in order to determine the position of them with a view to HSE. This descriptive-analytical study is being carried out based on the review of the literature and its results to identify the factors of HSE climate and then assessing the climate of HSE among the staff of a combined cycle power plant. The survey (questionnaire) contains forty-three questions and is adjusted based on the 9- point Likert Scale Eight factors are being determined by means of an appropriate correlation for assessing the HSE climate. The validity of the questionnaire was achieved by means of Cronbach's Alpha coefficient of 0.727 and the final result of the questionnaire evaluates an intermediate climate of HSE in the organization.

\section{INTRODUCTION}

Technologic achievements and rapid changes in evolved and precipitous world and increased risks, detrimental environment factors, for facilitating to the economic institutions proficiency, national economic growth and human, financial sources productivity; it is needed to select suitable approaches, strategies and ways and implement them. Because biological environment has restrictions in spite of best controls and technologies for developments. Also, economic development without environmental consideration losses it [1]. The countries which have reached institutional developments have benefited a principle and reasonable attitude in their constructed thinking. These countries pay special attention to the human and his needs and regard human resources as infrastructure development. In these countries privilege thinking and kind of attitude to the human are considered as the main base and infrastructure of development. Undoubtedly, achieving long -time goals in production alongside productivity of them requires providing demands of human forces and maintaining them against working risks and increasing his/her physical -mental health levels which are considered as keeping and maintaining resources and environment.

Based on world organizational statistic, in average 317 million employment accidents occurs per year, among them 2, 3 million people losses their lives. In fact, every second 153 worker involved in accidents in which one of them results to death (ILO-2017). Thus, the role of HSE management system as one of necessary management systems in each organization for recognition of HSE risks, sanitary and environmental aspects, adoption of approaches and planning for improving and solving problems are more important. In case of creating a sanitary, safety and positive health environmental culture, it would be efficient for achieving goals of stable developments As Trand Konshovich believes, HSE is base and foundation of everything, so that recognition its culture and understanding our wishes are more important and necessary [2].
HSE atmosphere is common perceptions of employers of HSE, their policies, approaches, functions. It's general importance and priority is in workplace. HSE atmosphere is a multidimensional factor and can be considered as main preface of HSE in work places. Measuring HSE atmosphere provides a snapshot picture of HSE status of organization in one discrete point of time. As it is multidimensional, also the picture specifies multidimensional of HSE status [3]. HSE atmosphere has direct effect on three cultural dimensions of safety, environmental / mental and behavioral status [4]. HSE atmosphere has different meanings in various cultural fields and often it is mistaken with HSE culture. HSE culture is part of an organization culture. HSE culture tends to have deep focus by less main values. It is easily available. The organizational hypotheses are about HSE and human resource [5]. Surely the HSE atmosphere strongly relates to HSE culture. So, HSE atmosphere is the perception importance of it by employees of an organization [6]. HSE atmosphere is defined as individual perceptions, procedures and related ways in workplaces [7]. HSE atmosphere is determining and predicating functions of it. HSE atmosphere affects the knowledge, incentives, complaints and partnerships of employees. Studies in regard to measuring HSE atmosphere are limit [8]

Thus, HSE atmosphere is complex of policies, approaches and rewards in relation to HSE problems that have perceived by employees. HSE atmosphere is special kind of organizational atmosphere which is describing personal perceptions of safety value in work place. These factors are as followings: management values, management proceedings, relationships and employee interventions in work place safety [9]. Organizational atmosphere is common perceptions among members of that organization by noting to aspects of environmental organization which informs behavioral role. The wide range of factors including management of values (such as management concerns for employees, their welfare), management and organizational behavior (e.g; education, providing safety equipment's, quality management systems of HSE), relationships and employee participation in work places as main components of HSE atmosphere have been recognized [10]. 
Many researchers have investigated HSE atmosphere constructions. But until Zoher offered HSE model in 1980, there were no agreement in relation to the number of factors needed for measuring HSE atmosphere or other effective factors [6].

HSE atmosphere has a lot of relationship with HSE culture. Meanwhile, its atmosphere emphasis that what conceptions of its importance workers have in the organization based on these common items on last definitions in order to get a general definition from HSE atmosphere" HSE atmosphere is considered as a scale of temporary situation of HSE culture and common perception of individual of that organization." Thus, it depends on place and time, also it refers to the perceived situation of HSE in special place and time, it is somehow unstable, and changes based on prevailing conditions [11]. Always there are different perceptions of HSE atmosphere between employees of various organizations and this difference could be considered as an evidence in different organizational culture of HSE and its situation, and management system efficiency [12].

HSE atmosphere is a multidimensional topic and it is addressed as indicator level of HSE in work places. Its measuring could be compared by evaluating "HSE heat" of another organization (in this way it will offer an immediate picture of HSE situation of an organization) [11]. According to studies, HSE atmosphere is of main indictors in occurring accidents and effecting management system of HSE. There are close relationships between increasing HSE atmosphere of an organization and decreasing accidents. So, paying attention to developing HSE policies in organizations and HSE atmosphere could be effective in improving HSE situation and decreasing accidents [13]. Employees' perceptions of HSE can be act as a mediator between a management system of HSE and causes decrease in accidents and promotion of HSE level in organization [14]. Also, employees by having positive perception of HSE situation in their own work place (positive HSE atmosphere) had accidents and registered less accidents.Thus,one good safety culture increase productivity and decrease expenses in long- time [15,16]. Different models have been applied for measuring safety atmospheres such as multi-level Zoher model, Neel and Grifeen safety measuring model, Felin safety atmosphere measuring model, Kristeen measuring model, Cooper and Filiphs atmosphere measuring model and so on [17].

In this paper, trying was on recognition of main factors and affecting indicators on measuring and evaluating HSE atmosphere of organization, in order to determine real position of HSE in one organization and follow implementing necessary planning for solving weak points and continues improvement of strength points of the complex (in this paper because of close meaning, in some places the word "safety" has defined to HSE). HSE climate is a multidimensional subject and is posed as an index of HSE Level in the places. Measuring HSE climate can be compared to evaluation of "HSE Heat" of an organization (which in this way it will provide a snapshot of HSE status of the organization) [11].

According to the conducted studies, HSE climate is among the crucial indices of events indication and effective in HSE Management System, and there is a close relationship between increase in organizational HSE climate and decrease of actions in the organization. Therefor; attention to development of HSE policies in the organizations and increase of HSE climate and culture in it can be fruitful in improvement of HSE status and decreasing the level of accidents [13]. Workers perception of HSE climate can act as a mediator amongst a HSE Management System and decrease the level of accidents and promote the level of organizational HSE [14].

Also, workers by positive perception of HSE status of their own workplace HSE (positive HSE climate) have had accidents and have registered less accidents. Thus, specifically a good safety culture will increase productivity and decrease the charges in the long run $[15,16]$.

For measuring the Safety climate of applied different models, such as multi-level of Zohar, Safety climate measuring model of Neal and Griffin, Safety climate measuring model of Flynn, Safety climate measuring model of Christian et al, Safety climate measuring model of Cooper, Philips and others [17].

In this article it has been tried that the crucial factors and effective indices in measuring and evaluation of organizational HSE climate to be identified, so that through them the real position of HSE is determined in an organization and on that basis, we can pursue the necessary executive programs to resolve weak points and constant improvement of strengths of the set. (because of closeness of meaning in this paper, in some cases the word safety has been defined in terms of HSE.)
In this descriptive-analytic research, initially the most important indices of HSE climate in the organization have been determined through studying and consideration of the former researches and evaluations, books and scientific references and referring to masters and experts and receiving their opinions and then the existing HSE climate amongst the staff of one of combinational cycle power plants has been performed. All of the Individuals' Aggregation (19 individuals) have been considered in this evaluation. To prepare questionnaire, 9 factors of management commitment, communications, HSE primacy, HSE laws and regulations, supportive environment, participation in HSE, personnel priorities and the need to HSE, personnel perception of the risk and work environment, have been evaluated in Likert Quintuple Range (Number 5 for absolute consent, Number 4 for consent, Number 3 indicating have no idea, Number 2 indicating against and number 1 indicating absolute against) and in the mold of 43 questions. The questionnaire was given to the individuals in their presence and the way to fill them out was explained to them. Before analysis of data, for considering the quality of data for analysis and examination of existence of relationship, KMO and Bartlett tests were utilized. Testing the evaluation of questionnaire was performed according to the following descriptions and testing the questionnaire stability and internal equal stability was determined by Cronbach's Alpha Coefficient. To consider the relationship between Safety climate factors with each other and also considering the relationship between demographic information with the total score of Safety climate, Spearman Correlation Coefficient has been utilized.

\subsection{Validity of questionnaire}

The concept of credit (validity) answers to this question that measuring tool to what extent evaluate the appointed characteristics. Without awareness of the measuring tools validity, the precision of emanated data of it cannot be relied upon. Measuring tools may be valid to measure a special feature, whilst for evaluation of the same feature on the other society, involve no validity [18].

\subsection{Test content validity consideration}

The first step in determining test validity, is content validity consideration. Content validity depends upon content logical analysis of a test and its determination is based on the mental and personal judgment. In this way, the test questions are put at discretion of experts or some of subjects and they are asked to specify that whether the test questions measure the appointed attribute or not and whether the questions include the total test content or not. In case that there is agreement between different individuals in the field of test validity, that test involves content validity. Content validity is two types, superficial and logical.

Considering the opinions of HSE masters and experts in terms of the questions, the required amendments for fluency of the sentences and expressions have been done as well.

A). CVR (content validity ratio): this index has been designed by a researcher [19]. For calculation of this index the opinions of experts in the field of the appointed test content is used and explaining test objectives for them and provision of operational definitions associated with test content to them, they are asked to classify each one of the questions on the basis of Likert Triple Range of "statement is necessary», "statement is helpful but not necessary» and «statement is not necessary».

Formula (1): CVR (Content Validity Ratio) calculation formula

$$
C V R=\frac{n_{E}-\frac{N}{2}}{\frac{N}{2}}
$$

B). CVI (Content Validity Index): in order to consider CVI (Content Validity Index) Waltz and Bausell method is used [20]. In such a way that the experts specify each statement «Relativity», «Clearance» and «Simplicity» based on a Likert quadripartite range. Experts specify the relativity of each statement in their own opinion from 1 «is not related», 2 «is rather related », 3 «is related» up to 4 «absolutely related». Simplicity of the statement also is specified respectively from 1 «is not simple», 2 «relatively is simple», 3 «is simple», up to 4 «is simple related» and clearance of the statement as well respectively from 1 «is not clear», 2 «is relatively clear», 3 «is clear» up to 4 «is clear related».

\section{MATERIALS AND METHODS}


Formula (2): CVI (Content Validity Index) calculation Formula

The Number of experts who have scored statements by $3 \& 4$

$\mathrm{CVI}-$

\section{Total Number of Experts}

The least acceptable amount for the index of CVI is equal to 0.79 and if CVI index of a statement is less than 0.79 , that statement must be omitted.

\subsection{Test superficial validity consideration}

In this method instead of attention to the test, the superficial characteristics of the test is noted and is set at the time when someone considers the test and conclude that this test measures the appointed attribute. This person can be a subject or an expert. Although this type of validity does not guarantee precise measurement, but with the motivation of the subject in answering, involves effect on validity of scores emanated from the test [21].

In order to consider the questionnaire validity, the opinions of 10 experts of HSE as for the questions one by one has been collected which had involved the necessary validity, furthermore CVI total questions equals with 0.93 .

\section{RESEARCH RESULTS}

In this research after summing up and determination of appointed indices, a questionnaire including 43 questions was prepared and distributed amongst all working personnel in a combinational cycle power plant. The individuals present in this evaluation had all BA and MA degrees and have a mean age range between 28 up to 37 years old.

The above-mentioned questionnaire stability was test with Cronbach's Alpha Coefficient test which have been indicated at an acceptable level and also the data possess a significant relationship with each other (the results of Bartlett and KMO tests have been shown in the table No. 1) [21].

Table 1: The results of Bartlett and KMO tests

\section{KMO and Bartlett's Test}

Kaiser-Meyer-Olkin Measure of Sampling Adequacy.

Bartlett's Test of Sphericity

Approx. Chi-Square
df
Sig.

In this sample the maximum mean of the scores are related to the priority of the personnel and the need to HSE and the least score is related to the personnel perception of the risk.

Table 2: Mean of Indexes Scores

\begin{tabular}{|c|c|c|}
\hline $\begin{array}{c}\text { Scores } \\
\text { Mean }\end{array}$ & Index & Row \\
\hline 8.0163158 & CommitmentManagement & 1 \\
\hline 8.0210526 & Communications & 2 \\
\hline 8.1315789 & PrimacyHSE & 3 \\
\hline 7.8878947 & Rules and RegulationsHSE & 4 \\
\hline 7.23 & EnvironmentSupportive & 5 \\
\hline 7.8984211 & Participation inHSE & 6 \\
\hline
\end{tabular}

\begin{tabular}{|c|c|c|}
\hline 8.6947368 & PrioritiespersonnelandNeed toHSE & 7 \\
\hline 5.7105263 & PerceptionpersonnelofRisk & 8 \\
\hline 7.2610526 & EnvironmentWork & 9 \\
\hline
\end{tabular}

For summing up the achieved results and ultimate results analysis of the organization HSE climate, the table (3) has been utilized.

Table (3): the procedure of calculating scores of indices

\begin{tabular}{|c|c|c|c|}
\hline $\begin{array}{l}\text { Strong } \\
\text { Climate }\end{array}$ & $\begin{array}{l}\text { Modest } \\
\text { Climate }\end{array}$ & $\begin{array}{c}\text { Weak } \\
\text { Climate }\end{array}$ & Index \\
\hline \multirow{9}{*}{$8 \leq X \leq 10$} & \multirow{9}{*}{$4 \leq X<8$} & \multirow{9}{*}{$2 \leq X<4$} & CommitmentManagement \\
\hline & & & Communications \\
\hline & & & PrimacyHSE \\
\hline & & & Rules and RegulationsHSE \\
\hline & & & EnvironmentSupportive \\
\hline & & & Participation inHSE \\
\hline & & & $\begin{array}{c}\text { PersonnelPrioritiesandNeed } \\
\text { toHSE }\end{array}$ \\
\hline & & & PerceptionpersonnelofRisk \\
\hline & & & EnvironmentWork \\
\hline $\begin{array}{c}72 \leq \mathrm{X} \leq \\
90\end{array}$ & $36 \leq X<72$ & $18 \leq X<36$ & ClimateTotal \\
\hline
\end{tabular}

Spearman Correlation Coefficient amongst HSE climate factors have been indicated in the table (4). There is a high significance level amongst most of HSE climate factors. The least relationship is in personnel perception of risk from the rest of indices and in fact it has had no convenient correlation.

Table 4: Spearman Correlation Coefficient amongst HSE Climate Factors

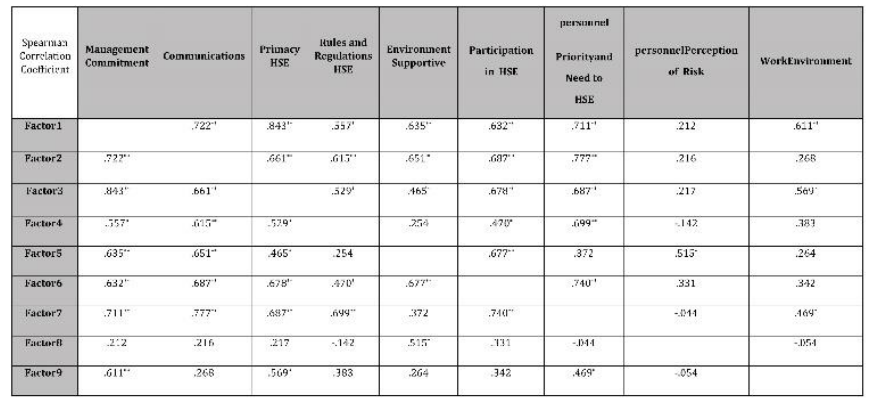

**. Correlation is significant at the 0.01 level (2-tailed).

*. Correlation is significant at the 0.05 level (2-tailed).

Also the total mean of HSE climate score is 68.9 which generally the organization HSE climate in the form of a general scheme (regarding the table 3) is evaluated as being modest.

\section{CONCLUSION}

Regarding the selection of 9 indices for consideration and evaluation of them in measuring organizational HSE climate and preparation of the related questionnaire, ultimately 8 index of management commitment, communications, HSE primacy, HSE rules and regulations, supportive environment, participation in HSE, personnel priorities and the need to HSE and work environment have been identified as the crucial indices in organization HSE climate.

In fact, with activity increase in each one of these domains, promotion of HSE climate can be acted upon and we can witness continuous 
improvement of Health, Safety and Environment.

Management commitment the first priority in HSE management system of each organization and the most important index in measuring HSE climate include the maximum relationship and correlation with other factors and planning in this section and its improvement will have a deserving effect in constant improvement of organizational HSE climate.

In the conducted case study, the scores have been related to the personnel perception of risk and regarding that the subject of risk is the most key discussion in HSE, it is necessary that the required planning to be executed for awareness of the personnel from risk and HSE culture promotion through that. Also, regarding the summed-up scores, the final score of HSE climate evaluation has been average and it is required that the necessary improvements to be done at the identified weak points.

\section{REFERENCES}

[1] Rezazadeh, S., Jahani, A., Makhdoum, M., and Meigooni, H.G. 2017. Evaluation of the Strategic Factors of the Management of Protected Areas Using SWOT Analysis-Case Study: Bashgol Protected Area-Qazvin Province. Open Journal of Ecology. 7, 55-68. http://www.scirp.org/journal/oje.

[2] Kongsvik, T., Gjøsund, G., Kristin, M., and Vikland. 2015. HSE culture in the petroleum industry: Lost in translation. Safety Science Page 5.

[3] Zohar, D. 2008. Safety climate and beyond: a multi-level multi-climate framework. Safety Science, 46, 376-387.

[4] Teo, E. A.-L., and Feng, Y. B. 2009. The Role of Safety Climate in Predicting Safety Culture on Construction Sites. Architectural Science Review, 52 (1), 5-16. http://dx.doi.org/10.3763/asre.2008.0037.

[5] Mearns, K.J., and Flin, R. 1999. Assessing the state of organization safety-culture or climate. Current Psychology, 18, 5-17.

[6] Lin, S.H., Tang, W.J., Miao, J.Y., Wang, Z.M., and Wang, P.X. 2008. Safety climate measurement at workplace in China: A validity and reliability assessment. Safety Science, 46, 1037- 1046.

[7] Neal, A., and Griffin, M. A. 2006. A Study of Lagged Relation- ships among Safety Climate, Safety Motivation, Safety Behavior, and Accidents at the Individual and Group Levels. Journal of Applied Psychology, 91 (4), 946-953. http://dx.doi.org/10.1037/0021-9010.91.4.946.

[8] Mansouriyeh, E.M.B., Khiyavi, M.F., Ravanbakhsh, M., Zadeh, M.H.H. 2013. Considering Safety climate of Ahvaz City rehabilitation clinics in the year 2013. safety promotion and prevention of injuries, 3 (1), 4-43.
[9] Nezhad, A.K., and Zia-oddini, M. 2015. Considering the relationship between safety climate and staff safety function regarding the mediating role of safety knowledge and motivation of safety in Mes-e

Sarcheshmeh complex. Quarterly of work health and safety, 5 (4), 69-86.

[10] Silva, S., Araújo, A., Dário Costa, J. L., Meliá . 2013. Safety Climates in Construction Industry: Understanding the Role of Construction Sites and Workgroups. 3, 80-86. http://dx.doi.org/10.4236/ojsst.2013.34010.

[11] Zeydi, E.M., Heydarniya, A., and Niknami, S. 2010. Evaluation of safety climate at the workplace: creation, validity and stability of questionnaire. Payesh quarterly, No. $10^{\text {th }}$ pages 157-166.

[12] Cooper, M. D., and Phillips, A. 2004. Exploratory Analysis of the Safety Climate and Safety Behavior Relationship. Journal of Safety Research, 35 (5), 497-512. http://dx.doi.org/10.1016/j.jsr.2004.08.004.

[13] Abbas, R.A., Zalat, M.M., Ghare. 2013. Non-Fatal Occupational Injuries and Safety Climate: A Cross-Sectional Study of Construction Building Workers in Mit-Ghamr City, Dakahlia Governorate, Egypt. Open Journal of $\begin{array}{llll}\text { Safety Science and Technology, 3, 69-79. } & \end{array}$ http://dx.doi.org/10.4236/ojsst.2013.34009.

[14] Jan, K., Wachter, Patrick, L., Yorio. 2014. The Mediating Role of Workers' Climate and Behavioral Perceptions on Safety Management System Performance. Open Journal of Safety Science and Technology, 4, 84-97. http://dx.doi.org/10.4236/ojsst.2014.42010.

[15] Smith, C., Silverman, G., Heckert, T. 2001. A Comprehensive Method for the Assessment of Industrial Injury Events. Journal of Prevention and Intervention in the Community, 22 (1), 5-20.

[16] Nielsen, K., and Mikkelsen, K. 2007. Predictive Factors for SelfReported Occupational Injuries at 3 Manufacturing Plants. Safety Science Monitor, 11 (2), 1-9.

[17] Heritage, Kyle, M. 2012. Ph.D [Lagged relationships between a multilevel model of safety climate and employee safety outcomes].Curtin University, School of Psychology and Speech Pathology.

[18] Lawshe, C.H. 1975. A quantitative approach to content validity. Personnel Psychology, 28, 563-575.

[19] Waltz, C. F., and Bausell, B. R. 1981. Nursing research: design statistics and computer analysis. New York: Davis FA.

[20] Naderi, E., and Naraghi, M.N.S, Badr, 1992. Research methods and the way of its evaluation in humanities sciences, Research and publication office, Tehran, fourth publish.

[21] Psychological test, Ali Fathi Ashtiyani, Tehran, Besat, 2010 